\section{La evidencia científica como motor de los procedimientos en Odontología}

\author{
Scientific evidence as the engine \\ of procedures in Dentistry
}

Jorge Luis Castillo Cevallos ${ }^{1, a}$

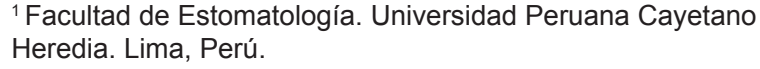

Fecha de recepción: 23/10/17

Fecha de aceptación: 31/10/17
Hace unos cuatro años la revista de la Asociación Americana de Ortodoncistas publicó un editorial, escrito por una de las figuras más importantes de la Ortodoncia contemporánea: el Dr Vincent Kokich. El título del editorial era " $¿$ Tiene usted una práctica clínica basada en evidencia?”. Dentro del artículo se podía leer una frase muy importante: "Una practica clínica basada en evidencia significa integrar la experiencia clínica individual con la mejor investigación clínica disponible" ${ }^{1}$.

¿Realmente practicamos eso? Formulemos un ejemplo. Llega a nuestro consultorio un paciente que ha terminado su tratamiento de Ortodoncia y presenta múltiples lesiones de mancha blanca. Nuestro objetivo es remineralizar esas lesiones iniciales a través de algún método que sea capaz de resolver el problema. Abrimos una página web y encontramos que existe una fórmula especial para remineralizar las lesiones iniciales. Para lograr el éxito usando ese sistema remineralizador hay que seguir una serie de pasos y el autor nos asegura que de esa manera las lesiones desaparecerán sin rastro. Nos entusiasmamos y nos preparamos para utilizar ese método mágico. Pero algo nos dice que debemos revisar con mayor profundidad la eficacia del método y decidimos buscar en la literatura científica. Al hacer la búsqueda, encontramos que según la ultima revisión sistemática del tema, publicada en el año 2017 por Hochli y colaboradores, las terapias con mejor evidencia para el manejo de las manchas blancas después del tratamiento de Ortodoncia son la pasta dental fluorada y la aplicación mensual de barniz de flúor (fluoruro de sodio al 5\%).
Este parece ser el mejor protocolo para reducir el área de la mancha blanca o aumentar la apariencia estética ${ }^{2}$.

¿Y dónde quedaron todas esas fórmulas mágicas para resolver las manchas blancas después del tratamiento de Ortodoncia? El no tener evidencia suficiente no quiere decir que no funcionen, pero hay que ser muy cuidadosos al utilizar métodos que sabemos no tienen un respaldo suficiente para afirmar que van a resolver el problema. Si existen métodos con un respaldo científico sólido, debemos apuntar a ellos sin dudarlo.

¿Hay alguna forma de hacer llegar al clínico general las últimas evidencias en los procedimientos clínicos de diagnóstico, prevención y tratamiento para que pueda utilizarlos en la práctica clínica diaria? Una de las maneras de llegar al clínico es a través de las Guías Clínicas Basadas en Evidencia. Estas Guías son documentos desarrollados sistemáticamente que tienen la intención de ayudar a los clínicos y pacientes a tomar decisiones acerca del cuidado apropiado de salud en circunstancias específicas. Las guías tienen el objetivo de mejorar la calidad de atención de los pacientes, promoviendo intervenciones que hayan demostrado ser de beneficio para el paciente, y descartando el uso de intervenciones inefectivas o potencialmente dañinas. Actualmente la biblioteca de la Red Internacional de Guías, tiene 6178 documentos de 76 países, que comprende guías para el diagnostico, prevención y tratamiento de diferentes condiciones y patologías humanas ${ }^{3}$.

Siendo la caries dental uno de los mayores problemas de salud pública en el Perú y otras partes del mundo y sien- 
do los niños la población más vulnerable, el Ministerio de Salud del Perú decidió hace más de 2 años iniciar el proceso de elaboración de la Guía de Práctica Clínica para el Diagnostico, Prevención y Tratamiento de Caries Dental en Niños ${ }^{4}$. Esta guía fue elaborada por los doctores Ailín Cabrera, Camila Palma y Jorge Luis Castillo. Fue un trabajo muy arduo y complejo, y finalmente se logró un producto que será de mucha utilidad para los clínicos y de gran beneficio para los pacientes.

La guía fue publicada oficialmente en mayo del 2017 y abarca tres áreas muy importantes relacionadas al manejo de la caries dental en niños: diagnóstico, prevención y tratamiento. Como ejemplo, en la sección de prevención, se resalta que las tres medidas preventivas con el más alto nivel de evidencia son: el uso de pasta dental fluorada de por lo menos $1000 \mathrm{ppm}$ a partir de la erupción del primer diente, la aplicación de barniz de flúor conteniendo fluoruro de sodio al 5\% y la aplicación de sellantes de fosas y fisuras.

Otras guías se están elaborando en este momento. Creemos que este es el camino correcto para lograr que los odontólogos peruanos manejen sus pacientes utilizando la mejor evidencia disponible. Es cierto que no todo ha sido estudiado, ni tampoco todo tiene un nivel de evidencia suficientemente alto. Pero debemos ser lo suficientemente capaces de integrar la mejor evidencia que tenemos a la mano (utilizando las guías de práctica clínica basadas en evidencia, o las revisiones sistemáticas más actualizadas) con la experiencia clínica utilizada de manera juiciosa y cuidadosa.

Lo que nos espera es el reto más grande: la implementación de las Guías. Sería un avance muy importante que todos los odontólogos puedan tratar a sus pacientes teniendo como referencia una guía clínica basada en evidencia científica. El proceso no es fácil y eso ha sido demostrado en otros países del mundo ${ }^{5}$. La obligación de todos los que conocemos la guía, es difundirla intensamente, porque eso va a permitir realizar procedimientos que generen el mayor beneficio en el paciente. $Y$ eso tiene un valor inmenso.

\section{Referencias bibliográficas}

1. Kokich VG. Do you have an evidence-based practice? Am J Orthod Dentofacial Orthop. 2013;143(1):1.

2. Höchli D, Hersberger-Zurfluh M, Papageorgiou SN, Eliades T. Interventions for orthodontically induced white spot lesions: a systematic review and meta-analysis. Eur J Orthod. 2017;39(2):122-33.

3. Murad MH. Clinical Practice Guidelines: A Primer on Development and Dissemination. Mayo Clin Proc. 2017;92(3):423-33.

4. Guía Ministerio de Salud del Perú. Guía de Práctica Clínica para la Prevención, Diagnóstico y Tratamiento de la Caries Dental en Niñas y Niños. Resolución Ministerial No 422-2017/MINSA. Agosto 23, 2017. [citado 30 de octubre 2017]. Disponible en: ftp://ftp2. minsa.gob.pe/normaslegales/2017/R.M._N\%C2\%B0_422-2017MINSA.pdf

5. Quanbeck A, Brown RT, E Zgierska A, A Johnson R, Robinson JM, Jacobson N. Systems consultation: protocol for a novel implementation strategy designed to promote evidence-based practice in primary care. Health Res Policy Syst. 2016;14:8. 